\title{
THE PATHOGENESIS OF INFECTIONS OF THE MOUSE CAUSED BY VIRULENT AND AVIRULENT VARIANTS OF AN INFLUENZA VIRUS
}

\author{
S. Raut,* J. Hurd,* R. J. Cureton, $\dagger$ G. Blandford $\ddagger$ \\ AND R. B. HEATH* \\ Departments of Virology* and Histopathology†, St Bartholomew's Hospital, London \\ and the Gage Research Institute $\ddagger$, University of Toronto, Canada

\section{Plates VII AND VIII}

INFECTION with respiratory viruses usually results in a mild transitory disease from which the host recovers. Our experience with Sendai-virus infection of the mouse suggests that the lack of severity of these infections is due to the induction of powerful defence mechanisms (Robinson, Cureton and Heath, 1968 and 1969; Blandford, Cureton and Heath, 1971). To obtain further information on these mechanisms, a virulent variant of a normally harmless influenza virus was obtained by serial passage in the lungs of mice. A comparative study was then made of the pathogenesis of the infections caused by this variant and the original avirulent virus.

\section{MATERIALS AND METHODS}

Virus. The Kunz strain of influenza-A virus was used, and was propagated in the allantoic cavity of 10-day-old embryonated hens' eggs. Dilutions of virus were prepared in broth saline for inoculation into mice and in medium 199 for inoculation into monkey kidney tissue cultures.

Infection of mice. Five-week-old CD1 mice were lightly anaesthetised and were infected by the intranasal inoculation of $0 \cdot 1-\mathrm{ml}$ volumes of virus suspensions. At various intervals after this, groups of six mice were weighed and were then exsanguinated under ether anaesthesia. Their lungs were removed, examined, weighed and then processed for histology or for determination of virus and interferon titres. The blood samples taken at these times were retained for assay of serum antibodies by HI. Lung lesions were scored by the method of Horsfall (1939).

Mouse-infectivity titres (MID50) were determined by inoculating serial 10-fold dilutions of virus into batches of ten mice. Deaths associated with typical pneumonitis and the presence of specific antibodies in the serum of mice surviving to the 14th day after inoculation were taken as evidence of infection. Titres were calculated by the method of Reed and Muench (1938).

Details of the histological and other assay procedures used have been given in previous papers (Robinson et al., 1968; Blandford et al., 1971; Blandford and Heath, 1972).

Immunofluorescence. Viral antigens and immunoglobulin-containing cells were detected by means of an indirect immunofluorescence technique as previously described (Blandford et al., 1971; Blandford and Heath, 1972 and 1974). A rabbit antiserum against Kunz virus was used for detecting viral antigens, and a rabbit anti-mouse-immunoglobulin serum for immunoglobulin-containing cells. 
Assay of non-specific inhibitors. The sensitivity of the viruses to inhibitors of haemagglutination present in normal mouse serum, and in $10 \%$ extracts of normal mouse lung in medium 199 was determined by the standard HI procedure in plastic microtitration plates.

Passage of virus in mice. A $10^{-3}$ dilution of allantoic fluid containing the original (M0) egg-adapted virus was inoculated intranasally into a group of four mice. Three days later their lungs were removed, homogenised in $4 \mathrm{ml}$ of medium 199 and a 1 in 10 dilution of the supernate was inoculated into a further batch of mice. This procedure was repeated 20 times. Virus present in the last lung extract was passaged twice in eggs to obtain seed (M20) for subsequent studies.

Identity of the passaged M20 virus. The haemagglutinins present in the M0 and M20 virus pools were shown to be antigenically identical by means of cross haemagglutinationinhibition tests on sera from mice infected with either virus.

A neutralisation test was performed in mice with a rabbit hyperimmune anti-M0 serum and the M20 virus. This serum prevented not only death of the animals but also infection, as judged by seroconversion, whereas the pre-inoculation serum from the same animal had no effect on either mortality or infection.

\section{RESULTS}

The Kunz influenza virus was successfully passaged in mice. A dose of $10^{3} \mathrm{TCD} 50$ of the M0 virus produced an apparently harmless infection after intranasal inoculation, whereas a comparable dose of the M20 virus induced a fatal pneumonitis in all or nearly all of the mice.

The respective haemagglutination titres, and the TCD50 and MID50 values are shown in table I. Passage did not increase the infectivity per unit of haemagglutinin of the virus for either tissue culture or mice.

Further experiments were made to obtain detailed comparative information on these two very different infections.

\section{Mortality resulting from the inoculation of various concentrations of the $M O$ and $M 20$ viruses}

To compare the lethal effects of various concentrations of the M0 and M20 viruses, pools of each were adjusted to contain the same number of tissueculture-infectious doses. Ten-fold dilutions of each pool were then prepared

\section{TABLE I}

Haemagglutination $(H A)$ titres and infectivity for tissue cultures (TCD50) and mice (MID50*) of the MO and M20 virus pools

\begin{tabular}{|c|c|c|c|c|c|}
\hline \multirow[b]{2}{*}{ Virus } & \multirow[b]{2}{*}{$\begin{array}{l}\log _{10} \\
\mathrm{HA} \\
\text { titre }\end{array}$} & \multicolumn{2}{|c|}{ Tissue-culture infectivity } & \multicolumn{2}{|c|}{ Mouse infectivity } \\
\hline & & $\begin{array}{l}\log _{10} \\
\text { TCD50 } \\
\text { per ml }\end{array}$ & $\begin{array}{l}\text { Ratio } \log _{10} \\
\text { TCD50: } \log _{10} \\
\text { HA titre }\end{array}$ & $\begin{array}{l}\log _{10} \\
\text { MID50 } \\
\text { per ml }\end{array}$ & $\begin{array}{l}\text { Ratio } \log _{10} \\
\text { MID50: } \log _{10} \\
\text { HA titre }\end{array}$ \\
\hline $\begin{array}{l}\text { MO } \\
\text { M20 }\end{array}$ & $\begin{array}{l}3 \cdot 1 \\
2 \cdot 8\end{array}$ & $\begin{array}{l}7.2 \\
6.9\end{array}$ & $\begin{array}{l}4 \cdot 1 \\
4 \cdot 1\end{array}$ & $\begin{array}{l}7.8 \\
7.2\end{array}$ & $\begin{array}{l}4 \cdot 7 \\
4 \cdot 4\end{array}$ \\
\hline
\end{tabular}

* See Materials and methods for definition. 
and inoculated into batches of 10 mice. Deaths associated with pneumonitis were recorded daily for 14 days and the results obtained are shown in fig. 1 . It can be seen that neither the M0 or M20 virus caused deaths when only 10 TCD50 of virus were inoculated. When inocula containing from $10^{2}$ to $10^{6}$ doses of the passaged M20 virus were used, virtually all of the mice died, and there appeared to be an inverse relationship between the dose of virus inoculated and the time of death after inoculation. In contrast to this, inocula of the M0 virus containing $10^{2}$ or $10^{3}$ TCD50 produced non-lethal infections. Higher doses of this virus $\left(10^{4}, 10^{5}\right.$ and $\left.10^{6} \mathrm{TCD} 50\right)$ caused some deaths but the mortality never exceeded $50 \%$.

In subsequent experiments, mice were given inoculations of exactly $10^{3}$ TCD50 of each virus, which consistently resulted in the M0 virus producing

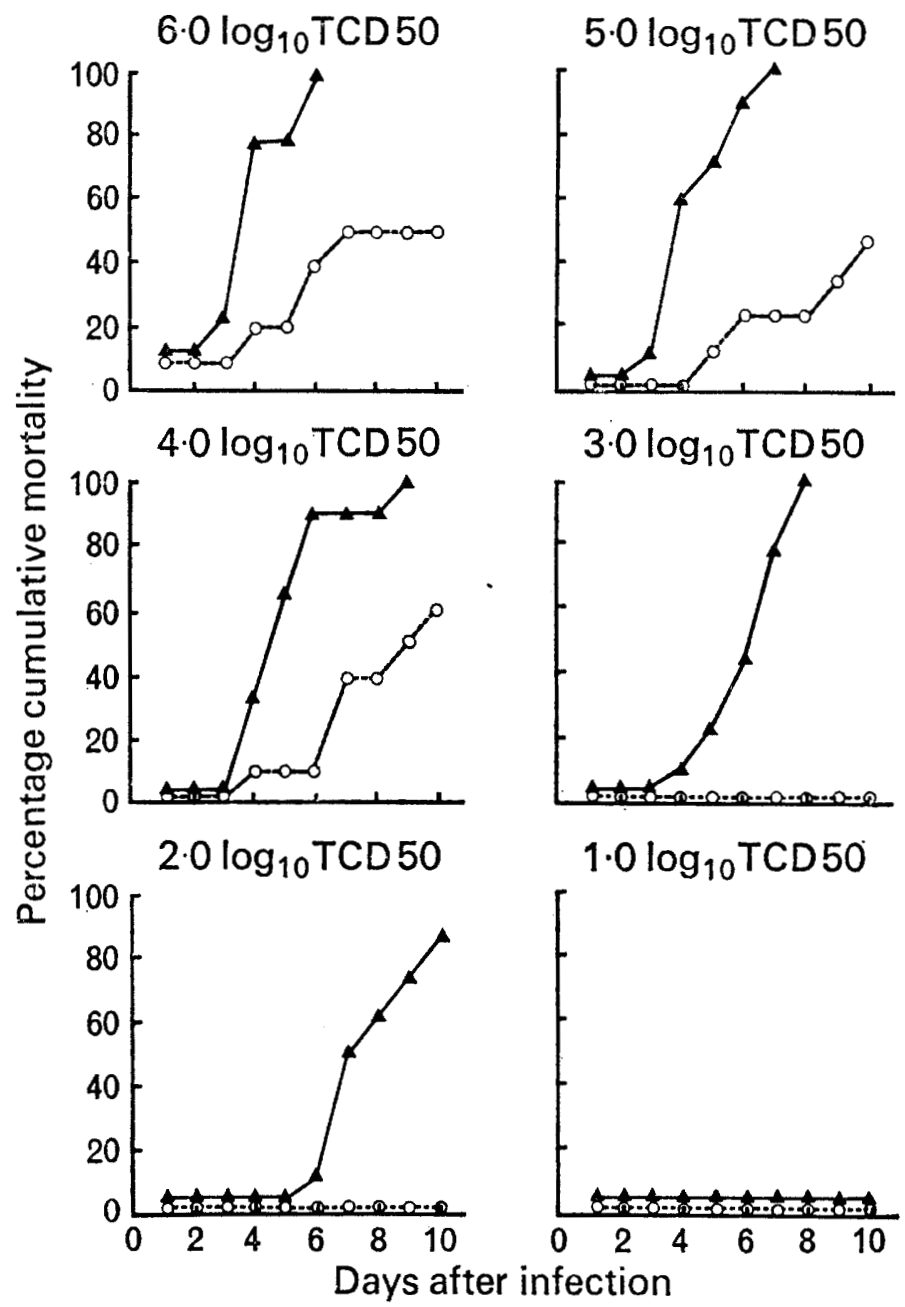

FIG. 1.-Cumulative mortality of mice infected with virus suspensions containing the indicated doses (TCD50) of M0 (O-O) or M20 (A ) virus. 
a mild infection from which all mice recovered, whereas all or nearly all of the mice infected with the M20 virus died with total lung consolidation. A series of three experiments of this kind were made during which a number of features of each infection were studied.

\section{General and local effects of the MO and M20 infections}

Some assessment of the general effects of the viruses on their hosts could be made by observing changes in total body weight during the course of the infections and the mean results in two of the experiments are shown in fig. 2. The difference in virulence between the two strains of viruses was apparent from the greater weight loss of the mice infected with the M20 than with the M0 virus.

Infection with either virus resulted in a comparable increase in lung weight during the first 3 days after inoculation, but thereafter the lungs of the M20infected mice were appreciably heavier than those of mice infected with the M0 virus. This excessive increase in weight of the lungs of the M20-infected mice was undoubtedly due to the development of consolidation, which was first noted at this time (see fig. 2). It will be noted that by the 5th day the lung score of the M20-infected mice was $2 \cdot 6$, indicating that, on average, over $60 \%$ of each lung was affected. Only trivial amounts of consolidation were seen in the lungs of mice infected with the M0 virus.
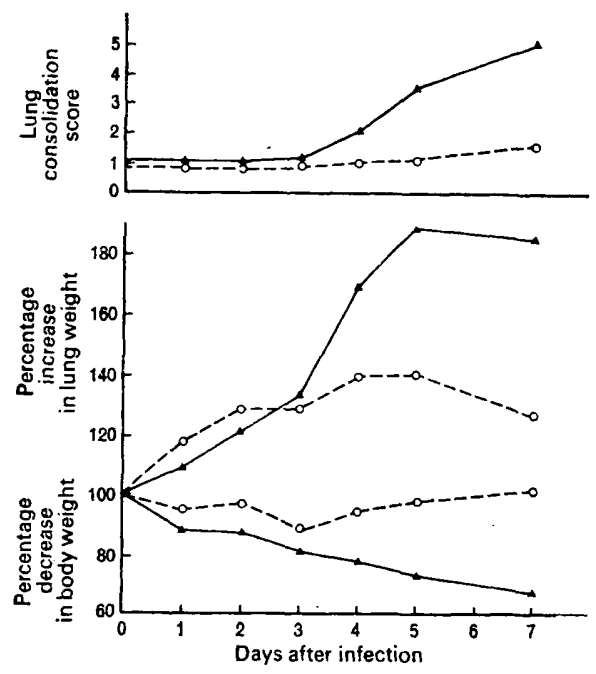

FIG. 2 (left).-The development of lung consolidation, increase in weight of lungs, and decrease in total body weight, after infection with M0 virus $(\mathrm{O}---\mathrm{O})$ and $\mathrm{M} 20$ virus $(\boldsymbol{\Delta}-\mathbf{\Delta})$.

Fig. 3 (right).-Virus growth, lung-interferon production and serum-antibody response in mice infected with the M0 virus $(\mathrm{O}-\ldots-\mathrm{O})$ and $\mathrm{M} 20$ virus $(\boldsymbol{A}-\mathbf{A})$. 


\section{Virus growth}

The growth of the M0 and M20 viruses in the lungs of the mice was determined in all three experiments (fig. 3). There was little difference between the initial growth rates of the two viruses and the peak titres they attained at 24-28 h. There was, however, a tendency for the M20 titres to be slightly the higher, and this was particularly apparent 6 and $9 \mathrm{~h}$ after inoculation. From the 3 rd day onwards there was a consistent and increasing difference between the growth curves, with the M20 virus again showing the higher titres.

It would appear unlikely that the increased virulence of the M20 virus was solely due to its slightly increased growth-rate during the first $24 \mathrm{~h}$ of the infection or to the slightly higher peak titres it attained. It is more likely that its virulence was in some way associated with the fact that it was better able to induce a more persistent infection of the lungs.

\section{Host-defence mechanisms}

The increased virulence of the Kunz virus after passage might have been due to the selection of mutants that were resistant to non-specific inhibitors present in body fluid or were poor inducers of interferon and antibody production.

The reactivity of the M0 and M20 virus to inhibitors present in normal mouse serum and in lung extracts of healthy mice was determined by haemagglutination-inhibition (table II). The M20 virus was slightly more resistant than the $\mathrm{M} 0$ virus to the inhibitors present in normal mouse serum, which was presumably $\beta$ inhibitor because it was heat labile. There was no difference in the sensitivities of the two strains to the inhibitors present in extracts of normal mouse lung. The activity in these extracts was presumed to be $\alpha$ inhibitor because it was heat stable but inactivated by receptor-destroying enzyme. The increased virulence of the M20 virus could not therefore be attributed to the development of resistance to non-specific inhibitors, which

TABLE II

Effect of non-specific inhibitors present in normal mouse serum and lung suspensions on the MO and M20 viruses

\begin{tabular}{l|cc}
\hline \multicolumn{1}{c|}{ Substance } & \multicolumn{2}{c}{$\begin{array}{c}\text { Titre of inhibitory activity of } \\
\text { the stated substance against }\end{array}$} \\
& $\overbrace{\text { MO virus }}$ & M20 virus \\
\hline Normal serum & 64 & 16 \\
Heated serum & $<8$ & $<8$ \\
Normal lung suspension & 40 & 40 \\
& $<5$ & $<5$ \\
\hline
\end{tabular}

* Receptor-destroying enzyme. 
could perhaps have been deduced from the finding that there was very little difference in the initial growth curves of the M0 and M20 viruses.

To study the effect of the virulent and avirulent viruses on the induction of host defence mechanisms, lung-interferon titres were determined in one and serum HI titres in all three of the experiments (fig. 3). The increased virulence of the M20 virus was clearly not due to its inability to induce or even depress these host functions, because it induced more interferon and an earlier and better serum-antibody response than did the avirulent $\mathrm{M} 0$ virus.

\section{Histological changes}

The earliest change-slight focal damage to the bronchial mucosa-could just be detected $12 \mathrm{~h}$ after infection with the M0 virus. This was more definite at $24 \mathrm{~h}$; the cytoplasm on the luminal side of the epithelial cells had a granular and ragged appearance and pyknotic nuclei, and a few polymorphonuclear leucocytes had appeared in the underlying submucosa. Lungs removed on the 2nd and 3rd days after infection showed increasingly widespread mucosal necrosis with debris lining the bronchial surfaces. The adjacent peribronchial and perivascular areas showed evidence of oedema and were infiltrated with predominantly polymorphonuclear leucocytes, which were seen marginating in nearby blood vessels. The changes were similar on day 4 , but mononuclear cells were now more dominant in the submucosal infiltrates.

From day 5 to day 7, signs of recovery from the bronchial infection were apparent. Exudate in the lumen had begun to clear and the inflammatory cellular cuffs were less striking. Mononuclear cells were now predominant in these cuffs and Unna-Pappenheim staining showed that many of these were pyroninophilic.

Considerable regeneration of the mucosa had occurred by the 8th day, and in some areas the earliest signs of exaggerated regeneration could be seen. The latter progressed during the next week, and in some areas had the appearance of small " tumourlets". Squamous metaplasia could be seen in other parts of the mucosa at this time.

The changes in the bronchial mucosa of the mice infected with the virulent M20 virus were essentially the same as those just described, but observations were of necessity restricted to the first 5 days of the infection because of the large number of deaths that occurred immediately after this. There were however subtle differences between the changes induced by the two viruses. For example, in mice infected with the M20 virus, there was a tendency for the focal lesions in the lung to be slightly more frequent and intense, for subsequent damage to the bronchial mucosa to be greater and more persistent, and for the mononuclear-cell response in the submucosa to develop rather more slowly than in mice infected with the M0 virus. The mucosal changes induced by these Kunz strains developed more rapidly than did the changes seen in mice infected with Sendai virus (Robinson et al., 1968) but were essentially similar in all other respects.

As was expected from the macroscopic appearances, the most profound 
differences between the histological changes induced by the two viruses were in the alveoli. The avirulent M0 virus induced minor changes in this tissue that could just be detected on the 3rd day, with a slight spread of the inflammatory response from the peribronchial region to the adjacent lungs; the alveolar walls had become more prominent and cellular, there was slight oedema and a few phagocytic cells were present in the alveolar spaces. By the 7th day, these spaces contained large foamy macrophages. These small areas of alveolitis persisted throughout the observation period but never became extensive.

Changes in the alveoli of the M20-infected mice were also first seen on the 3rd day after infection but even at this early stage these were more pronounced than those in the M0-infected lungs. The differences between the two infections can best be seen on the 4th day (see figs. $4 a$ and $b$ ). The alveolitis in the lungs of the mice infected with the M20 virus spread rapidly during the course of the infection, so that by the 5 th and 7 th days virtually all the lung fields were affected.

\section{Immunofluorescence studies}

Immunofluorescence staining was used to detect the localisation of viral replication in different parts of the lungs removed at various times after infection. At $12 \mathrm{~h}$, viral antigens were detected in scattered areas of the bronchial mucosa of the lungs of mice infected with both the M0 and M20 viruses. During the next few days the staining spread rapidly to other areas of mucosa and increased in intensity. M20 viral antigen was always slightly more widely distributed through more bronchi than was the M0 antigen. Viral antigens could not be detected in the lungs of mice infected with the M0 virus after the 8th day but similar observations could not be carried out on the M20 infected mice because of the large number of deaths that occurred from the 6th day onwards.

The most striking difference between the distribution of viral antigen in the lungs of the mice infected with the two viruses was in the alveoli. Appreciable amounts of viral antigen were detected in alveolar cells as early as $12 \mathrm{~h}$ after infection with the virulent M20 virus, and an increasing number of these cells became infected during the course of the infection (fig. $5 b$ ). It appeared that alveolar cells and macrophages were supporting viral replication but the latter requires further investigation. Similar events occurred with the M0 virus infection (fig. $5 a$ ) but were later, less frequent and much less extensive.

From day 2, sections that had been eluted with alkaline buffer before being stained for viral antigens showed increased amounts of staining in comparison with untreated parallel sections. This suggested that a local antibody response had occurred with both viruses at about the same time (Blandford and Heath, 1972).

Studies for the appearance of immunoglobulin-containing cells revealed a sub-mucosal influx of such cells by $24 \mathrm{~h}$. There was a suggestion that more immunoglobulin containing cells appeared in the bronchial sub-mucosa of M0- than of M20-infected mice. The numbers of these cells increased with time until the end of the experiments. The most striking difference between 
M0 and M20 infections again occurred in the lung parenchyma. Wherever there was virus some immunoglobulin-containing cells could usually be found nearby. In the areas of consolidation, hundreds of such cells could be seen, together with diffuse extracellular fluorescence indicating aggregated accumulations of immunoglobulin (figs. $6 a$ and $b$ ).

\section{DisCUSSION}

It has frequently been observed that serial passage of influenza viruses in the mouse lung results in the appearance of variants with the ability to produce extensive and fatal pneumonitis. It has been suggested that passage results in the selection of virulent mutants present in the original virus pool (Davenport 1954; Ledinko 1956). The increased virulence of the adapted strains has been attributed to their lack of sensitivity to $\beta$ inhibitors (Briody, Cassel and Medill, 1955 ) and to their ability to attain higher titres in lung tissue (Sugg, 1950). The latter was not, however, a property of the strains studied by Hirst (1947).

The Kunz strain of influenza virus was easily adapted to mice by serial passage of lung extracts. The passaged virus differed from the original eggadapted strain in that inoculation of low doses consistently resulted in fatal pneumonic diseases. Both the original and the adapted strains had the same infectivity per unit of haemagglutinin for tissue cultures and for mice as judged by seroconversion.

Passage did not result in the virulent strain acquiring appreciable resistance to non-specific inhibitors, and it was also found that the passaged strain induced more interferon and a more rapid appearance of serum antibody than the original virus. The elution studies suggested that local antibody production started at the same time in both infections, and large numbers of immunoglobulin-containing cells were present in the lung parenchyma of the M20 virus infected mice. It is clear therefore that the virulence of the passaged strain was not due to its loss of immunogenicity or to any depressing effect on immune responses.

The adapted strain grew slightly faster and attained slightly higher titres than the original virus during the first $24 \mathrm{~h}$ of the infection. This finding was confirmed by immunofluorescence staining for viral antigens and by the observation that this strain produced slightly more damage to the mucosa during the early stages of the infection. It is, however, unlikely that the differences observed were alone sufficient to account for the increased virulence of the adapted strain.

The most significant difference between the two viruses revealed by these studies was the greater ability of the passaged strain to infect alveolar cells. This was directly demonstrated by immunofluorescence staining for viral antigens and was suggested by the more extensive development of pneumonitis in the early stages of the infection. This altered pathogenesis might have been brought about by the slightly increased mucosal growth of the passaged strain facilitating extension of the infection to the lung parenchyma or by altered tropism of this strain. 


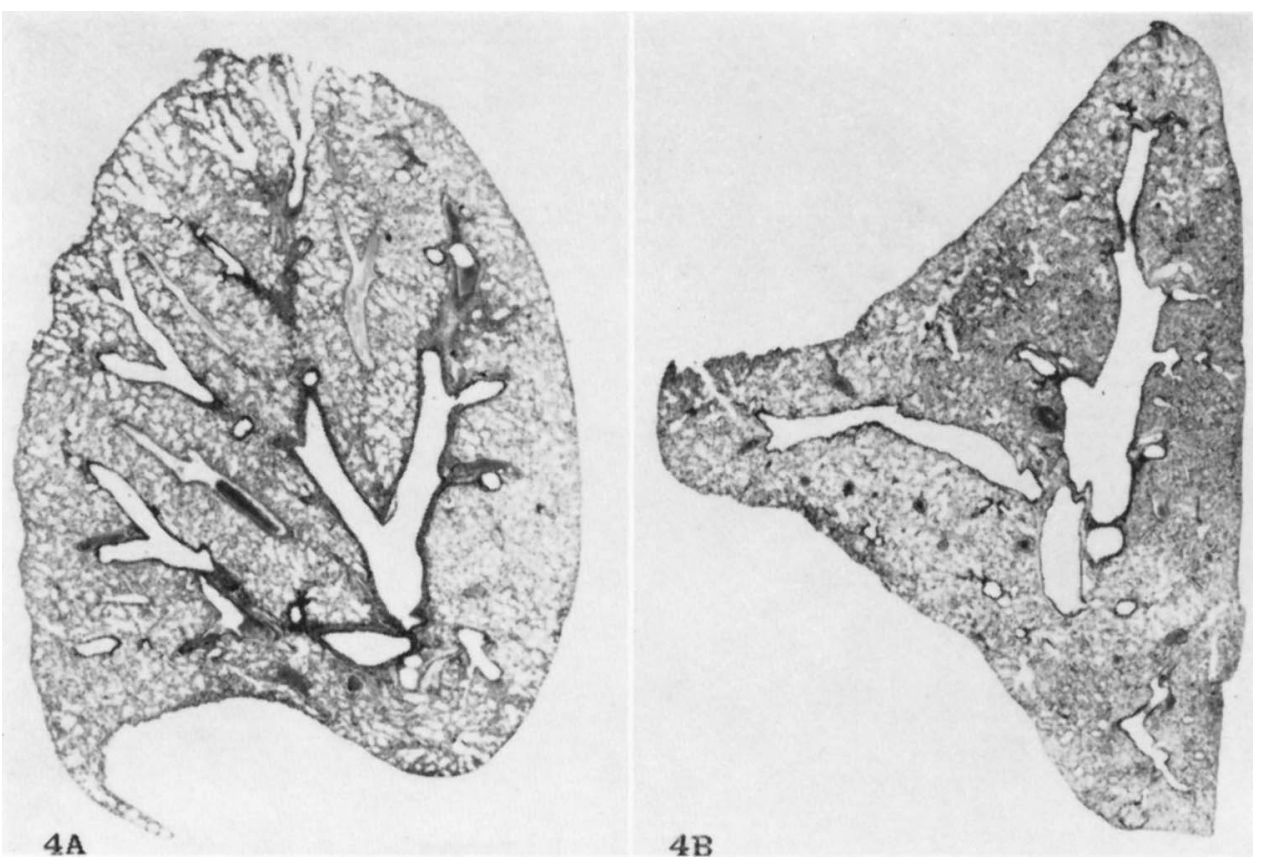

Fig. 4.-Sections of lungs removed on the 4th day after inoculation (a) with M0 virus: Note some small areas of alveolitis extending from the inflamed bronchi; $(b)$ with M20 virus, with more widespread alveolitis, Haematoxylin and eosin. $\times 10$. 

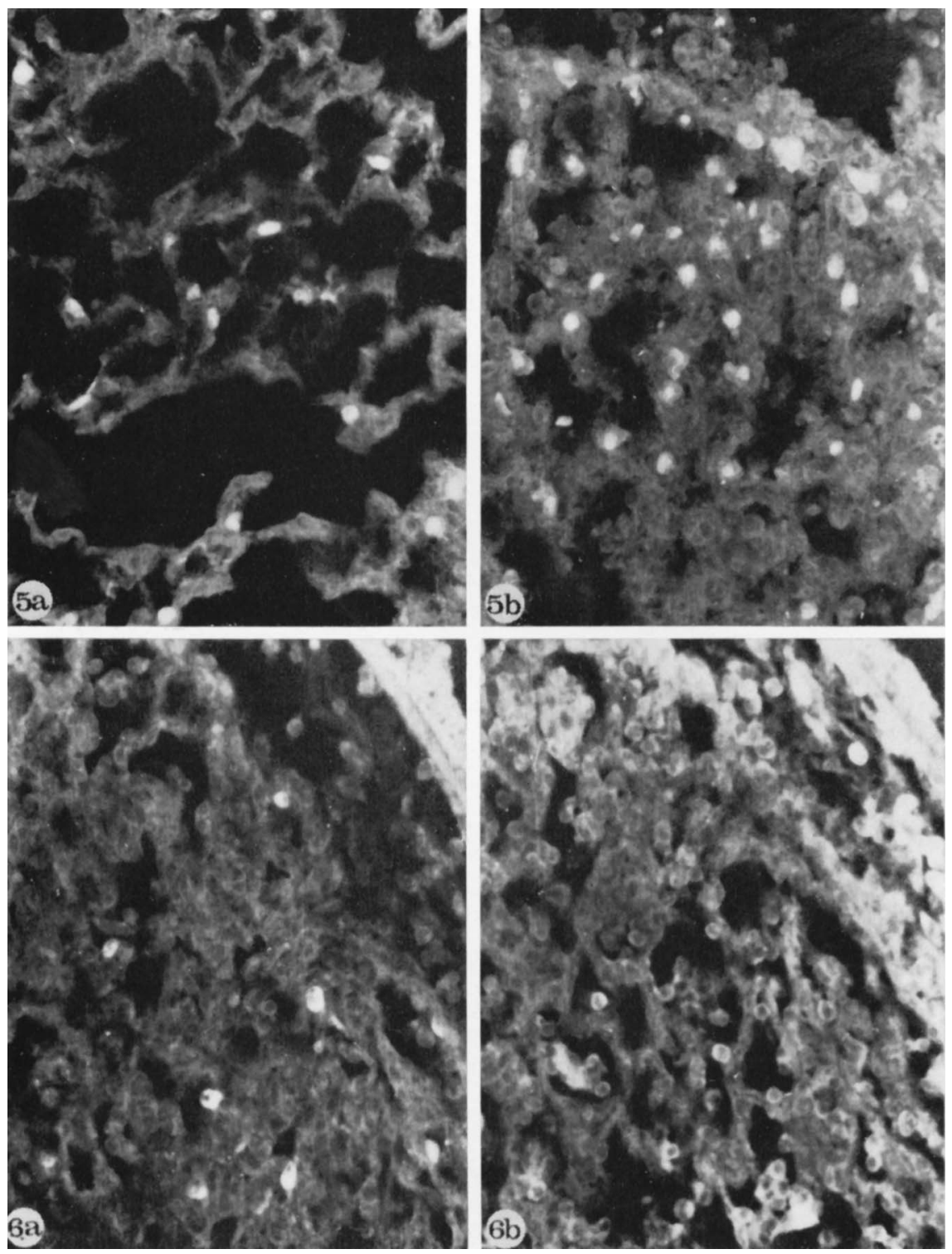

FIG. 5.-Immunofluorescence staining for viral antigens on the 2 nd day after infection: (a) M0 infection, showing scanty involvement of alveolar cells; (b) M20 infection with more extensive alveolitis affecting almost the whole of this lobe. $\times 250$.

FIG. 6.-(a) M20-infected lung at day 2 stained for viral antigens. Note the presence of antigen in numerous alveolar cells and in the bronchial mucosa seen in the upper right hand corner. $(b)$ Parallel section to $(a)$ but stained for immunoglobulins. Note the numerous positive-staining cells in the lung parenchyma and a collection in the bronchial sub-mucosa. $\times 250$. 
It is not immediately apparent why the immune mechanisms appear to provide a very effective defence for infection restricted to the mucous membrane but are inadequate when the infection involves alveolar cells. This may be related in some way to the fact that the mice had more difficulty in ridding themselves of the M20 virus. Before this can be understood it will be necessary to undertake more detailed comparative studies of these mechanisms in the submucosa and lung parenchyma.

It seems likely that the dramatic difference between the two infections is simply due to varying tolerance of inflammatory changes in different parts of the lung. Immediate or rapid involvement of numerous alveoli and the ensuing inflammatory changes result in such serious loss of respiratory function that death ensues before the immune response can become effective. Comparable inflammatory changes that are restricted to the submucosa and lumen of the airway, such as are induced by the non-passaged virus, are not immediately life-threatening, and this allows time for the immune response to eradicate the virus and for recovery to occur.

\section{SUMMARY}

A virulent variant of the normally avirulent Kunz strain of influenza virus was obtained by serial passage in mice, and the pathogenesis of the infections caused by the two strains was studied.

The virulence of the passaged variant did not appear to result from increased growth in lungs, from acquisition of resistance to non-specific inhibitors or from inadequate immunogenicity, but from its greater ability to replicate in alveolar cells.

The apparent adequacy of defence mechanisms to protect against mucosal infection but their failure to protect against infection of alveolar cells is discussed.

We wish to thank the Nuffield Foundation and the Canadian Medical Research Council (Grant no. MA-5019) for their financial support for this work.

\section{REFERENCES}

Blandford, G., Cureton, R. J. R. AND Heath, R. B. 1971. Studies of the immune response in Sendai virus infection of mice. J. med. Microbiol., 4, 351.

BlandFord, G. AND Heath, R. B. 1972. Studies on the immune response and pathogenesis of Sendai virus infection of mice. I. The fate of viral antigens. Immunology, 22, 637.

BlandFoRd, G. AND HEATH, R. B. 1974. Studies on the immune response and pathogenesis of Sendai virus infection of mice. II. The immunoglobulin class of plasma cells in the bronchial sub-mucosa. Immunology, 26, 667.

Briody, B. A., Cassel, W. A. ANd Medill, M. A. 1955. Adaptation of influenza virus to mice. 3. Development of resistance to $\beta$ inhibitor. J. Immunol., 74, 41.

DAVENPORT, F. M. 1954. The inequality of potential in influenza virus for adaptation to mice. J. Immun., 72, 485 .

HIRST, G. K. 1947. Studies on the mechanism of adaptation of influenza virus to mice. J. exp. Med., 86, 357.

Horsfal, F. L. 1939. Neutralization of epidemic influenza virus. The linear relationship between the quantity of serum and the quantity of virus neutralized. J. exp. Med., 70, 209. 
LEDINKo, N. 1956. An analysis of the process of adaptation of influenza virus B of recent human origin to the mouse lung. J. gen. Microbiol., 15, 47.

ReED, L. J. AND MuenCH, H. 1938. A simple method of estimating $50 \%$ end point. Am.J. Hyg., 27, 493.

Robinson, T. W. E., Cureton, R. J. R. and Heath, R. B. 1968. The pathogenesis of Sendai virus infection in the mouse lung. J. med. Microbiol., 1, 89.

Robinson, T. W. E., Cureton, R. J. R. and Heath, R. B. 1969. The effect of cyclophosphamide on Sendai virus infection of mice. J. med. Microbiol., 2, 137.

SUGG, J. Y. 1950. The relation of the concentration of unadapted and adapted influenza virus in the mouse lung to the death or survival of the infected host. J. Bact., 60, 489. 